arXiv:1603.04491

\title{
Cerenkov-Like Emission of Pions by Photons in a Lorentz-Violating Theory
}

\author{
Brett Altschul] \\ Department of Physics and Astronomy \\ University of South Carolina \\ Columbia, SC 29208
}

\begin{abstract}
In the presence of Lorentz violation, the Cerenkov-like process $\gamma \rightarrow \gamma+\pi^{0}$ may become allowed for sufficiently energetic photons. Photons above the threshold would lose energy rapidly through pion emission. The fact that propagating photons with energies of up to $80 \mathrm{TeV}$ survive to be observed on Earth allows us to place a one-sided constraint on an isotropic Lorentz violating parameter at the $7 \times 10^{-13}$ level; this is more than an order of magnitude better than the best previous result.
\end{abstract}

\footnotetext{
${ }^{1}$ baltschu@physics.sc.edu
} 
One interesting possibility for new physics beyond the standard model is that there might be violations of Lorentz and CPT symmetries. So far, these are strictly theoretical possibilities; there has been no compelling evidence to suggest that these symmetries are not truly exact in nature. However, if these symmetries turn out not to hold exactly, that would certainly be a discovery of great importance. The discovery would open up a window onto a completely new regime of physics.

The fundamental physical theories that we currently understand - the standard model and general relativity - are both fully invariant under Lorentz symmetry and CPT. However, the machinery of effective field theory can be used to describe modified versions of these theories that do not respect the symmetries exactly. The most general effective field theory that is used to describe such symmetry violations is known as the standard model extension (SME). The SME action contains all the possible translation-invariant but Lorentz-violating operators that could be constructed out of standard boson and fermion fields. The operators that have residual tensor indices violate Lorentz symmetry, and they may break CPT symmetry as well. The coupling constants of the theory are vector and tensor valued; their Lorentz indices are contracted with the free indices found on the field operators [1, 2]. If the origin of the Lorentz violation is spontaneous symmetry breaking, these tensors, which represent preferred directions in spacetime, are related to the vacuum expectation values of dynamical vector and tensor fields.

One of the virtues of the SME is its generality; it contains operators of arbitrarily high mass dimension. However, a specific restricted version of the SME has become the standard framework for parameterizing the results of most empirical Lorentz and CPT tests. The restricted version is known as the minimal SME, and it contains only local, gauge-invariant operators of renormalizable mass dimension. With these restrictions in place, the minimal SME contains only a finite number of undetermined parameters, and radiative corrections are systematically calculable [3, 4, 5]. This makes the minimal SME framework well suited for comparing the results of different experiments, and experiments in many different areas of physics have been used to constrain the minimal SME coefficients. The best current constraints are summarized in [6].

Many of the tightest constraints on the Lorentz-violating operators of the SME come from analyses of astrophysical data. Two particular things are available in extraterrestrial environments that can make astrophysical tests of Lorentz symmetry extremely sensitive: very large distances and very high energies. Observations of photons that have traversed cosmological distances can be exquisitely sensitive to the tiniest changes in electromagnetic wave propagation. The strongest constraints on other effects come from looking at the emissions from extremely energetic astronomical sources, in which individual particles may have energies at up to the $\mathrm{PeV}$ scale.

By studying the photons emitted by such sources, we may learn a great deal about the energy-momentum relations not just for the photons themselves, but other particles as well. The observation of a photon with a TeV-scale energy coming from an extraterrestrial source can tell us things in two different ways. If the process that is responsible for 
the photon emission at the source is understood, the energies of the emitted photons can reveal important information about the energy-momentum relations for the other particles involved in the emission process. The typical processes that produce ultrahigh-energy $\gamma$-rays are inverse Compton scattering, $e^{-}+\gamma \rightarrow e^{-}+\gamma$ (in which a lowenergy photon is upscattered by an extremely energetic electron); and neutral pion decay, $\pi^{0} \rightarrow \gamma+\gamma$. When we observe photons that we know to originate from one or the other of these processes, we learn a substantial amount about the energy-momentum relations for the massive particles involved. This has made it possible to place strong constraints on Lorentz violation in the electron sector of the SME [7, 8, 9] and somewhat weaker constraints in the pion sector [10].

Moreover, observations of cosmic ray $\gamma$-rays can also tell us interesting things about Lorentz violation in the electron and pion sectors, even when the processes by which the photons were produced are unknown. The reason is that, while photons in the Lorentzinvariant standard model are absolutely stable in vacuum, sufficiently energetic photons may actually decay if there is Lorentz violation present. For a photon from a distant source to survive long enough to reach Earth, photon decay processes such as pair creation, $\gamma \rightarrow e^{-}+e^{+}$, or Cerenkov-like pion emission, $\gamma \rightarrow \gamma+\pi^{0}$, must be forbidden (or exceedingly slow) at the observed photon energy. This provides a complementary way of constraining Lorentz violation outside the photon sector using $\gamma$-ray observations.

Previously, the survival of $\mathrm{TeV}$ photons has been used to place complementary constraints on electron-sector Lorentz violation. The fact that these photons live long enough to be seen ensures that $\gamma \rightarrow e^{-}+e^{+}$is not occurring [11]. Of course, an electron and a positron are not the only particle pair that might be produced in such a reaction. The fact that photons do not decay via $\gamma \rightarrow \pi^{+}+\pi^{-}$allows us to place bounds on the SME coefficients for charged pions [12. These bounds are weaker than the electron-sector bounds, because the typical strength of a bound derived from a high-energy astrophysical process is $\sim m^{2} / E^{2}$, where $m$ is the mass of the heaviest particle involved in the process and $E$ is the energy scale of the process. Yet while this means that the constraints in the charged pion sector are orders of magnitude worse that the equivalent constraints in the electron sector, the pion bounds are still important. The reason is that bounds on pion Lorentz violation are typically rather difficult to obtain. In fact, it is generally true that the constraints on Lorentz violation for unstable species are frequently quite weak, and the goal of this paper will be to improve the constraints on Lorentz violation for the even shorter lived neutral pion.

Lorentz violation in the SME is described by tensor-valued coefficients contracted with tensor operators constructed out of the particle fields. The minimal SME Lagrange density for the free neutral pion field is

$$
\mathcal{L}_{\pi}=\frac{1}{2}\left(\partial^{\mu} \pi^{0}\right)\left(\partial_{\mu} \pi^{0}\right)+\frac{1}{2} k^{\mu \nu}\left(\partial_{\mu} \pi^{0}\right)\left(\partial_{\nu} \pi^{0}\right)-\frac{m_{\pi}^{2}}{2}\left(\pi^{0}\right)^{2} .
$$

The Lorentz violation in the pure pion sector is governed by the nine small coefficients contained in the traceless, symmetric $k^{\mu \nu}$ tensor. These coefficients, which appear in the 
action for the composite pion field, must ultimately be related to the SME coefficients for the more fundamental quark and gluon fields.

The pions are coupled to photons, and the Lagrange density for the pure photon sector and the pion-photon coupling is

$$
\mathcal{L}_{A}=-\frac{1}{4} F^{\mu \nu} F_{\mu \nu}-\frac{1}{4} k_{F}^{\mu \nu \rho \sigma} F_{\mu \nu} F_{\rho \sigma}+\frac{1}{2} k_{A F}^{\mu} \epsilon_{\mu \nu \rho \sigma} F^{\nu \rho} A^{\sigma}-g \pi^{0} \epsilon^{\mu \nu \rho \sigma} F_{\mu \nu} F_{\rho \sigma} .
$$

The electromagnetic action may also contain Lorentz violation, governed by the $k_{F}$ and $k_{A F}$ coefficients. However, we shall consider only theories with vanishing $k_{F}=0$ and $k_{A F}=0$. Photon Lorentz violation is comparatively easy to constrain; some of the coefficients have been bounded extremely tightly using cosmological searches for photon birefringence [13, 14, 15], while the remainder have been bounded at a far less stringent (but still quite respectable) level using direct Michelson-Morley tests of the isotropy of photon propagation [16. In contrast, bounds on the SME coefficients for unstable massive particles like the $\pi^{0}$ are much weaker. So we shall focus on the sensitivity of various observables to the less well constrained pion $k^{\mu \nu}$ parameters.

The key to using photon observations to constrain $\pi^{0}$ Lorentz violation is, of course, the pion-photon coupling. The coupling, which is normally responsible for the $\pi^{0} \rightarrow \gamma+\gamma$ decay, is dominated by the chiral anomaly $g \approx \frac{N_{c} e^{2}}{96 \pi^{2} f_{\pi}}$, where $N_{c}=3$ is the number of colors and $f_{\pi}$ the pion decay constant. In a Lorentz-violating theory, the Cerenkov-like process $\gamma \rightarrow \gamma+\pi^{0}$, which is ordinarily forbidden by energy-momentum conservation, may become allowed above a certain energy threshold.

The Lorentz-violating dispersion relation for an ultrarelativistic particle with mass $m$ typically takes the form

$$
E=\sqrt{m^{2}+[1+2 \delta(\hat{p})] \vec{p}^{2}} .
$$

The parameter $\delta(\hat{p})$ determines the maximum achievable velocity (MAV) for the particle type in question. The MAV $1+\delta$ depends on the direction $\hat{p}$ of the momentum (and for a fermionic particles, $\delta$ would also depend on the helicity). In general, $\delta$ is a function of the dimensionless coefficients multiplying the dimension-four operators in the relevant sector of the minimal SME. There may be additional terms in the energy-momentum relation derived from dimension-three operators, but their importance diminishes with increasing energies, and so they have relatively little impact on highly relativistic processes.

For the $\pi^{0}$ the MAV is set by

$$
\delta(\hat{p})=-\frac{1}{2}\left[k_{00}+k_{(0 j)} \hat{p}_{j}+k_{j k} \hat{p}_{j} \hat{p}_{k}\right],
$$

where $k_{(0 j)}=k_{0 j}+k_{j 0}$. With observations of photons coming from a sufficient number of different directions, it could be possible to place separate bounds on all nine of the $k^{\mu \nu}$ parameters. However, we shall restrict our attention to the case of an isotropic theory with constant $\delta=-\frac{2}{3} k_{00}$ (taking into account the tracelessness of $k^{\mu \nu}$ ). 
We shall now look in detail at how the $\gamma \rightarrow \gamma+\pi^{0}$ process behaves in the presence of a nonzero pion $\delta$. The most obvious fact is that the process can only occur if $\delta<0$. In the standard theory, the photon carries insufficient energy to create the pion. However, with a negative $\delta$, the pion will have less energy than it would in the standard theory at the same momentum. Although a low-energy photon will still not have sufficient energy to emit a pion, the Cerenkov-like emission will become allowed above a certain photon threshold energy.

The kinematics of the pion emission are fairly straightforward. The initial photon has momentum $q^{\mu}=(E, 0,0, E)$. The outgoing photon loses energy and is deflected by an angle $\theta$; it carries momentum $q^{\prime \mu}=\left(E^{\prime}, E^{\prime} \sin \theta, 0, E^{\prime} \cos \theta\right)$. This leaves the pion carrying momentum $p^{\mu}=\left(E_{\pi},-E^{\prime} \sin \theta, 0, E-E^{\prime} \cos \theta\right)$. The pion energy-momentum relation (3) dictates that

$$
\left(E-E^{\prime}\right)^{2}=E_{\pi}^{2}=m_{\pi}^{2}+\left(E^{2}+E^{\prime 2}-2 E E^{\prime} \cos \theta\right)(1+2 \delta),
$$

so the relationship among the various quantities can be expressed

$$
\sin ^{2}(\theta / 2)=-(1-2 \delta) \frac{m_{\pi}^{2}}{4 E E^{\prime}}-\delta \frac{\left(E-E^{\prime}\right)^{2}}{2 E E^{\prime}} .
$$

The pion emission process can only occur above the threshold energy $E_{T}=\frac{m_{\pi}}{\sqrt{-2 \delta}}$. If $\delta$ is negative, then for sufficiently large $E>E_{T}$, the Cerenkov-like emission becomes possible. In the threshold configuration, the pion carries away all the energy, and the outgoing photon energy $E^{\prime}$ is zero. Well above threshold, a typical decay will have the pion and photon each carrying off a substantial fraction of the energy, since decays with comparable $E^{\prime}$ and $E_{\pi}$ have the largest available phase space. In other words, the outgoing particles are usually beamed into a narrow pencil of angles.

However, decays with large photon deflection angles are still possible, even though they are strongly disfavored. In a decay with a vanishing deflection angle $\theta$, the photon carries an energy $E^{\prime}=E-\sqrt{E_{T}^{2}+m_{\pi}^{2}}$, which is obviously the majority of the energy if the initial energy $E$ is well above the threshold. At the other extreme, a photon with energy $E^{\prime}=m_{\pi}^{2} / E$ will recoil back with angle $\theta=\pi$ after the decay.

The matrix element for the process is quite straightforward to calculate. The Feynman rule for the pion-photon-photon vertex, derived from the Lagrange density (2), has a factor 8ig $\epsilon^{\alpha \mu \beta \nu} q_{1 \alpha} q_{2 \beta}$, where $q_{1}$ and $q_{2}$ are the photon momenta directed into the vertex. The matrix element for the Cerenkov-like process is then

$$
i \mathcal{M}=8 i g \epsilon^{\alpha \mu \beta \nu} q_{\alpha}\left(-q_{\beta}^{\prime}\right) \varepsilon_{\mu}(q) \varepsilon_{\nu}^{*}\left(q^{\prime}\right),
$$

where the $\varepsilon$ are the appropriate polarization vectors. So the matrix element squared (summed over final polarizations) for the process is

$$
\begin{aligned}
\sum|\mathcal{M}|^{2} & =32 g^{2} \epsilon^{\alpha \mu \beta \nu} \epsilon^{\gamma \rho \delta \sigma} q_{\alpha} q_{\gamma} q_{\beta}^{\prime} q_{\delta}^{\prime} g_{\mu \rho} g_{\nu \sigma} \\
& =-64 g^{2}\left[q^{2} q^{2}-\left(q \cdot q^{\prime}\right)^{2}\right] \\
& =256 g^{2} E^{2} E^{\prime 2} \sin ^{4} \theta .
\end{aligned}
$$


The fact that the matrix element $\mathcal{M}$ is proportional to $\theta^{2}$ for small deflection angles has a relatively straightforward explanation, tied to the involvement of the totally antisymmetric Levi-Civita $\epsilon$-tensor. The initial photon has four-momentum $q^{\mu}=(E, 0,0, E)$, and after the emission, the outgoing photon has $q^{\prime \mu}=\left(E^{\prime}, E^{\prime} \sin \theta, 0, E^{\prime} \cos \theta\right)$. For small values of $\theta, E^{\prime} \approx E-p_{3}$, and so

$$
q^{\prime \mu} \approx\left(E-p_{3}, 0,0, E-p_{3}\right)+\left(0,-p_{1}, 0,0\right) .
$$

The vectors $q$ and $q^{\prime}$ are both contracted with an $\epsilon$-tensor. The first term on the righthand side of (11) therefore makes no contribution, because it is proportional to $q^{\mu}$. This leaves the whole matrix element proportional to $-p_{1}=E^{\prime} \sin \theta$.

This accounts for the presence of one factor of $\sin \theta$. The second factor arises in a similar fashion from the contraction of the incoming and outgoing polarization vectors with the $\epsilon$-tensor. The possible polarization vectors for the incoming photon are $\varepsilon_{1}=$ $(0,1,0,0)$ and $\varepsilon_{2}=(0,0,1,0)$. However, only the polarization vector $\varepsilon_{2}$ pointing along the $y$-direction can occur for the kinematics we are considering; only if the initial polarization is along the $y$-direction, will the photon be deflected in the $x z$-plane. The reason is that $\varepsilon_{1}$ is (approximately) a linear combination of $q$ and $q^{\prime}$ [as given by (11)], and thus there cannot be a nonvanishing contribution when all three vectors are contracted with a common $\epsilon$-tensor.

For the outgoing photon, there are also two possible polarization vectors. One of them is the unchanged, out-of-plane $\varepsilon_{2}$. However, this vector is obviously impossible, since one factor of $\varepsilon_{2}$ must already be contracted with the $\epsilon$-tensor. Therefore, the outgoing polarization must be $\varepsilon_{1}^{\prime} \approx(0, \cos \theta, 0,-\sin \theta)$, which is the other unit vector perpendicular to $\vec{q}^{\prime}$; and only the $z$-component of this $\varepsilon_{1}^{\prime}$ contributes to the contraction, providing the second factor of $\sin \theta$ in $\mathcal{M}$.

For the conventional decay $\pi^{0} \rightarrow \gamma+\gamma$, there is an analogous $\theta^{2}$ suppression factor in $\mathcal{M}$ when the angle between the decay photons is small. The derivation of this factor follows along essentially the same lines in that case. The fact that the small-angle behaviors of the matrix elements for the two decays are similar will enable us to estimate the $\gamma \rightarrow \gamma+\pi^{0}$ rate from the well-known $\pi^{0} \rightarrow \gamma+\gamma$ rate.

Of course, the rate for the novel Cerenkov-like process can be evaluated directly from the matrix element and the Lorentz-violating kinematics. However, the kinematical part of the calculation turns out to be extremely awkward. Instead, it is possible to estimate the rate using what is known about the rate for the ordinary two-photon decay of the $\pi^{0}$. This might initially seem problematic, since the pion lifetime is normally calculated in the pion's rest frame, and the parent photon in $\gamma \rightarrow \gamma+\pi^{0}$ has no equivalent rest frame. However, an unstable particle's decay rate can certainly be calculated in a frame in which the initial particle is moving, and we know that in a Lorentz-invariant theory, the decay rate will be diminished (relative to the rest frame rate) by the Lorentz factor $\gamma$. Moreover, it is actually fairly straightforward how this factor arises in the calculation. In the frame in which the parent particle is moving, the decay products are preferentially 
emitted along the direction of that motion. In an ultrarelativistic process, the decay products are beamed into a narrow pencil of angles, of characteristic size $\sim m / E$ (where $m$ and $E$ are the mass and energy of the parent particle), rather than covering the full $4 \pi$ steradians. This limits the phase space available for the decay, and the rate is reduced by precisely the time dilation factor $\gamma^{-1}=m / E$.

The same kind of dilation occurs in the photon decays that may be allowed in Lorentzviolating theories. While the parent particle in $\gamma \rightarrow \gamma+\pi^{0}$ has no rest frame, the daughter particles are overwhelmingly bunched into an angular range $\sim m_{\pi} / E_{T}$. The characteristic lifetime for a single particle decaying into two ultrarelativistic daughters, when the interaction is governed by the anomaly term from (2), is given by the $\pi^{0}$ lifetime of $\tau_{\pi} \approx 8.4 \times 10^{-17}$ s. So except very close to threshold, the rate of the $\gamma \rightarrow \gamma+\pi^{0}$ is $\Gamma \sim m_{\pi} / E_{T} \tau_{\pi}$.

The $\theta^{4}$ suppression of the $|\mathcal{M}|^{2}$ at small angles does not befuddle this result, since the same suppression is present in the ordinary $\pi^{0} \rightarrow \gamma+\gamma$ process. However, there is a puzzling aspect to this. With increasingly relativistic energies, the decay photons (in the $\pi^{0}$ decay) are beamed into a narrowing pencil of angles, but in spite of the $\theta^{4}$ factor in $|\mathcal{M}|^{2}$, the rate decreases only quite slowly. (The time dilation makes the rate proportional to a single power of the separation angle $\theta$.) Of course, the change in the decay rate with energy is entirely dictated by Lorentz symmetry in this case. The resolution of this puzzle is that the matrix element also grows with energy as $|\mathcal{M}| \propto E^{4}$, and $E^{4} \theta^{4} \propto E^{4}\left(m_{\pi} / E\right)^{4}$ is independent of the energy. The same characteristic argument applies in the Lorentzviolating Cerenkov-like photon decay process.

If the transit time for a photon of energy $E>\alpha E_{T}$ from its source to the Earth is much longer than $\sqrt{|\delta|} \tau_{\pi}$, the photon will lose most of its energy through Cerenkov-like pion emission on its journey. The factor $\alpha$ is present to ensure that the energy is not too close to threshold; right above threshold, the pion emission angle falls to zero, and the decay time differs significantly from $\sim \sqrt{|\delta|} \tau_{\pi}$. For the specific observations we shall consider below, $\alpha=1.01$ turns out to be more than sufficient.

Conversely, the observation of a photon from a sufficiently distant source indicates that its energy $E$ must be less than $\alpha E_{T}$; otherwise it would not have survived. This places a bound on $\delta$ :

$$
\delta>-\frac{\alpha^{2} m_{\pi}^{2}}{2 E^{2}}
$$

Note that even for a $1 \mathrm{PeV}$ photon, $E \tau_{\pi} / m_{\pi} \sim 10^{-9}$ s, so effectively any astrophysical source is distant enough that the observation of an emitted photon should produce a reliable bound. Since the $\gamma$-ray spectrum of the Crab nebula extends up to at least 80 $\mathrm{TeV}$ [17], we can conclude that

$$
\delta>-7 \times 10^{-13} .
$$

This represents an improvement of more than an order of magnitude over the best previous bound. 
The best previous constraint on a negative $\delta$ for the $\pi^{0}$ field came from observations of $\gamma$-rays that were known to originate from the $\pi^{0} \rightarrow \gamma+\gamma$ process. That was a major limitation, since many of the highest-energy $\gamma$-rays appear to be produced by inverse Compton scattering instead. The current method is much more widely applicable. The survival of any $\gamma$-ray, regardless of how it originated, over long astrophysical distances, allows us to place a useful bound. The bound derived from this technique is the strongest presently available, since it is be based on the very highest energy $\gamma$-ray observations. In the not-so-distant future, it may be possible to observe photons with PeV energies (since there is already evidence of individual particles with PeV energies in the Crab nebula [18]); that would lead to another two orders of magnitude improvement in the constraint (12).

So the observed absence of photon decay processes gives a powerful way to constrain Lorentz violation. However, this method does have a drawback. Any photon decay process is generally only going to become allowed if the particles produced in the decay have less energy than they would in the standard Lorentz-violating theory. In other words, only negative values of $\delta$ can be constrained this way. This also makes it difficult to study more general theories, in which the Lorentz violation includes anisotropy as well as boost invariance violation. The observation of photons coming from different directions would allow us to place bounds on the $\delta(\hat{p})$ parameters corresponding to different directions. However, with only one-sided bounds, it is not possible to disentangle these bounds to get bounds on the individual $k^{\mu \nu}$ coefficients. The only available bounds on a positive $\delta$ for the $\pi^{0}$ are at the $2 \times 10^{-9}$ level, and this limits how tightly the individual $k^{\mu \nu}$ parameters may be constrained.

Ultimately, the best future constraints on Lorentz violation in the $\pi^{0}$ sector may come from a clearer understanding of the relationships between the fundamental SME coefficients for the quark and gluon fields and the coefficients for composite fields like the pion. However, at present, the best bounds on dimension-four Lorentz-violating operators for the $\pi^{0}$ field come from observations of $\mathrm{TeV} \gamma$-rays. In this paper, we have given an improved one-sided constraint, derived from the observed absence of the Cerenkov-like emission process $\gamma \rightarrow \gamma+\pi^{0}$ for Crab nebula $\gamma$-rays with up to $80 \mathrm{TeV}$ energies. This represents an order of magnitude improvement over the best previous bounds.

\section{References}

[1] D. Colladay, V. A. Kostelecký, Phys. Rev. D 55, 6760 (1997).

[2] D. Colladay, V. A. Kostelecký, Phys. Rev. D 58, 116002 (1998).

[3] V. A. Kostelecký, C. D. Lane, A. G. Pickering, Phys. Rev. D 65, 056006 (2002).

[4] D. Colladay, P. McDonald, Phys. Rev. D, 75, 105002 (2007).

[5] A. Ferrero, B. Altschul, Phys. Rev. D 84, 065030 (2011). 
[6] V. A. Kostelecký, N. Russell, Rev. Mod. Phys. 83, 11 (2011); updated as arXiv:0801.0287v9.

[7] T. Jacobson, S. Liberati, D. Mattingly, Nature 424, 1019 (2003).

[8] B. Altschul, Phys. Rev. Lett. 96, 201101 (2006).

[9] B. Altschul, Phys. Rev. D 75, 041301 (R) (2007).

[10] B. Altschul, Phys. Rev. D 77, 105018 (2008).

[11] F. W. Stecker, S. L. Glashow, Astropart. Phys. 16, 97 (2001).

[12] B. Altschul, Astropart. Phys. 28, 380 (2007).

[13] S. M. Carroll, G. B. Field, R. Jackiw, Phys. Rev. D 41, 1231 (1990).

[14] V. A. Kostelecký, M. Mewes, Phys. Rev. Lett. 97, 140401 (2006).

[15] V. A. Kostelecký, M. Mewes, Phys. Rev. Lett. 99, 011601 (2007).

[16] M. Nagel, S. R. Parker, E. V. Kovalchuk, P. L. Stanwix, J. G. Hartnet, E. N. Ivanonv, A. Peters, M. E. Tobar, Nature Comm. 6, 8174 (2015).

[17] F. Aharonian, et al., Astrophys. J. 614, 897 (2004).

[18] F. A. Aharonian, A. M. Atoyan, in Neutron Stars and Pulsars, edited by N. Shibazaki, N. Kawai, S. Shibata, T. Kifune (Universal Academy Press, Tokyo, 1998), p. 439 astro-ph/9803091. 